\title{
Identification of potential pathogenic biomarkers in clear cell renal cell carcinoma
}

\author{
ZENGZENG WANG $^{1,2}$, ZHIHONG ZHANG $^{1}$, CHANGWEN ZHANG $^{1}$ and YONG XU ${ }^{1}$ \\ ${ }^{1}$ Department of Urology, Tianjin Institute of Urology, The Second Hospital of Tianjin Medical University, \\ Tianjin 300211; ${ }^{2}$ Department of Urology, Tianjin Beichen Hospital, Tianjin 300400, P.R. China
}

Received September 15, 2017; Accepted February 16, 2018

DOI: $10.3892 / \mathrm{ol} .2018 .8398$

\begin{abstract}
The purpose of the present study was to screen potential pathogenic biomarkers of clear cell renal cell carcinoma (ccRCC) via microarray analysis. The mRNA and microRNA (miRNA) expression profiles of GSE96574 and GSE71302 were downloaded from the Gene Expression Omnibus (GEO) database, as well as the methylation profile of GSE61441. A total of 5 ccRCC tissue samples and 5 normal kidney tissue samples were contained in each profile of GSE96574 and GSE71302, and 46 ccRCC tissue samples and 46 normal kidney tissue samples were involved in GSE61441. The differentially expressed genes (DEGs) and the differentially expressed miRNAs (DEMs) were obtained via limma package in ccRCC tissues compared with normal kidney tissues. The Two Sample t-test and the Beta distribution test were used to identify the differentially methylated sites (DMSs). The Database for Annotation, Visualization and Integrated Discovery (DAVID) was used to perform the Gene Ontology (GO) term and Kyoto Encyclopedia of Genes and Genomes (KEGG) pathway enrichment analyses of the DEGs. The targets of the DEMs were screened with the miRWalk database, and the further combination analyses of DEGs, DEMs and DMSs were conducted. Additionally, reverse transcription PCR (RT-PCR) and methylation-specific PCR (MS-PCR) were performed to detect the mRNA level and methylation status of HAPLN1. The mRNA levels of $h s a-m i R-204$ and $h s a-m i R-218$ were tested by RT-PCR. A total of 2,172 DEGs, 202 DEMs and 2,172 DMSs were identified in RCC samples compared with normal samples. The DEGs were enriched in 1,015 GO terms and 69 KEGG pathways. A total of 10,601 miRNA-gene pairs were identified in at least 5 algorithms of the miRWalk database. A total of 143 overlaps were identified between the
\end{abstract}

Correspondence to: Dr Yong Xu, Department of Urology, Tianjin Institute of Urology, The Second Hospital of Tianjin Medical University, 23 Ping-Jiang Road, He Xi, Tianjin 300211, P.R. China E-mail: xuyonguro@163.com

Key words: clear cell renal cell carcinoma, pathogenesis, differentially expressed genes, differentially expressed microRNAs, differentially methylated sites
DEGs and the differentially methylated genes. Furthermore, the DEGs were involved in 851 miRNA-gene pairs, including 127 pairs in which the target genes were negatively associated with their corresponding DEMs and DMSs. HAPLNI was lowly expressed and highly methylated in ccRCC tissues, while $h s a-m i R-204$ and $h s a-m i R-218$ were highly expressed. The results of the present study indicated that HAPLN1, $h s a-m i R-204$ and $h s a-m i R-218$ may be involved in the pathogenesis of ccRCC.

\section{Introduction}

Renal cell carcinoma (RCC) is the most common type of kidney cancer responsible for $90-95 \%$ of all cases, and accounting for $\sim 3 \%$ of adult malignancies (1). Clear cell RCC (ccRCC) is the most aggressive RCC subtype and constitutes $70-80 \%$ of all RCC cases with the highest rates of local invasion, metastasis and mortality (2). RCC is usually asymptomatic in the early stages and, as the disease progresses, signs include hematuria, flank pain, abdominal masses and loin pain (3). An unhealthy lifestyle is a major cause of RCC, and it has been reported that smoking, obesity and hypertension have been estimated to cause $\sim 50 \%$ of all cases (4). Additionally, hereditary factors have an impact on individual susceptibility to RCC (5). Other genetically-linked conditions also increase the risk of developing RCC, including hereditary papillary renal carcinoma, hereditary leiomyomatosis, hyperparathyroidism-jaw tumor syndrome, familial papillary thyroid carcinoma and sickle cell disease. The pathogenesis of RCC is extremely complex and is yet to be elucidated. Notably, an increasing number of biomarkers have been found to be involved in the pathogenesis of RCC. Matsuura et al (6) proved that the downregulation of $S A V I$ and the consequent YAPl activation were involved in the pathogenesis of high-grade ccRCC. Furthermore, bioinformatics analyses demonstrated that microRNAs (miRNAs) were dysregulated in ccRCC and may contribute to kidney cancer pathogenesis by targeting more than 1 key molecule (7). A larger number of miRNAs are associated with key pathogenesis mechanisms of hypoxia and epithelial-to-mesenchymal transition, including $m i R-200, m i R-210, m i R-155, m i R-8 a$, $m i R-424, m i R-381, m i R-34 a, m i R-17-5 p$ and $m i R-224$ (8). In addition, promoter region methylation and transcriptional silencing are major mechanisms of tumor suppressor genes 
in RCC (9). Ricketts et al (10) reported that certain tumor suppressor genes were methylated in RCC tumor tissue (e.g., SLC34A2 was specifically methylated in $63 \%$ of RCC cases, OVOL1 in 40\%, DLEC1 in 20\%, TMPRSS2 in $26 \%$, SST in $31 \%$ and BMP4 in $35 \%$ ). Therefore, the methylation analysis is an attractive strategy for investigating novel genes in the pathogenesis of RCC. In the present study article, an mRNA expression profile, a miRNA expression profile and a methylation profile of ccRCC were synthetically analyzed in order to screen potential pathogenic biomarkers via microarray analysis.

\section{Materials and methods}

Microarray data. The microarray datasets of GSE96574, GSE71302 (11) and GSE61441 (12) were downloaded from the Gene Expression Omnibus (GEO) database (www.ncbi.nlm. nih.gov/geo/). GSE96574, which was an mRNA expression profile with 5 ccRCC tissues and 5 normal kidney tissues, was detected with the platform of Agilent-067406 CBC IncRNA + mRNA microarray V4.0; GSE71302, an miRNA expression profile with 5 ccRCC tissues and 5 normal kidney tissues, was detected with the platform of Agilent-021827 Human miRNA Microarray V3; GSE61441, a methylation profile with $46 \mathrm{ccRCC}$ tissues and 46 normal kidney tissues, was detected with the platform of Illumina HumanMethylation450 BeadChip.

Data processing and differential analysis. For the profiles of GSE96574, GSE71302 and GSE61441, the raw data were obtained and normalized using the preprocess core function package V3.5 (http://www.bioconductor.org/packages/release/ bioc/html/preprocessCore.html) (13). Subsequently, the differentially expressed genes (DEGs) and differentially expressed miRNAs (DEMs) were identified in ccRCC samples compared with normal kidney samples with the limma V3.18.13 software package (http://www.bioconductor.org/packages/ 2.13/bioc/html/limma.html). $\mathrm{P}<0.05$ and $\mid \log _{2}$ (fold-change) $\mid>1$ were used as threshold criteria. The two sample t-test and the $\beta$ distribution test were used to identify the differentially methylated sites (DMSs), and DMSs were identified with $\mathrm{P}<0.05$ and $|\Delta \beta|>0.2$. Furthermore, the genes in which the DMSs were located were labeled using the annotation files of the methylation chip platform.

Functional and pathway enrichment analysis of DEGs. Gene Ontology (GO) terms and Kyoto Encyclopedia of Genes and Genomes (KEGG) pathway enrichment analyses of DEGs were performed via the Database for Annotation, Visualization and Integrated Discovery (DAVID) V6.8 (http://david.abcc.ncifcrf.gov/) (14). GO terms and KEGG pathways were selected with $\mathrm{P}<0.05$.

Target prediction of DEMs. To investigate the related regulation mechanisms of DEMs, the targets and their locations were predicted by the miRWalk V2.0 database (http://www.umm.uni -heidelberg.de/apps/zmf/mirwalk/), which was a powerful and accurate database that displayed miRNAs, their corresponding target genes and binding sites in mice, rats and humans (15). Putative targets were predicted by $>5$ bioinformatics algorithms among the 10 algorithms in the miRWalk database: DIANAmT V4.0 (www.ma.uni-heidelberg.de/apps/ zmf/mirwalk/diana-microt), miRanda -rel2010 (www.ma .uni-heidelberg.de/apps/zmf/mirwalk/miranada), miRDB V4.0 (www.ma.uni-heidelberg.de/apps/zmf $/ \mathrm{mirwalk} / \mathrm{mirdb}$ ), miRWalk V2.0 (www.ma.uni-heidelberg.de/apps/zmf/mirwalk /mirwalk), RNAhybrid V2.1 (www.ma.uni-heidelberg.de/apps/ zmf/mirwalk/rnahybrid), PICTAR4 (www.ma.uni-heidelberg.de/apps/zmf/mirwalk/pictar4), PICTAR5 (www.ma .uni-heidelberg.de/apps/zmf/mirwalk/pictar5), PITA (www .ma.uni-heidelberg.de/apps/zmf/mirwalk/pipa), RNA22 V2 (www.ma.uni-heidelberg.de/apps/zmf/mirwalk/ rna22) and Targetscan V6.2 (www.ma.uni-heidelberg. de/apps/zmf/mirwalk/targetscan). Therefore, the miRNA-gene regulation pairs were screened out and the locations of the targets were drawn out.

Combination analysis of DEGs, DEMs and DMSs. The corresponding genes of DMSs were identified based on the $\beta$-value. If multiple DMSs corresponded to a single gene, the average $\beta$-value of the DMSs was used as the $\beta$-value of the gene. The overlapped genes between the DEGs and the corresponding genes of DMSs were screened out with the threshold of $|\Delta \beta|>0.2$. The genes involved in the aforementioned miRNA-gene pairs and the DEGs were selected out and further analyzed with their corresponding DEMs and DMSs.

Verification of associated genes and miRNAs in patients with ccRCC. A total of 10 patients with ccRCC, 32-57 years old (mean age, 63.2), were collected between February 2017 and March 2017, including 5 male patients and 5 female patients. The tumor tissues and adjacent non-cancerous tissues were collected with surgical resection. Written informed consent was obtained when the patients were accepted by the Second Hospital of Tianjin Medical University. All procedures were performed in accordance with the ethical standards of the institutional and/or national research committee. The total RNA was extracted using TRIzol (Invitrogen; Thermo Fisher Scientific, Inc., Waltham, MA, USA). Reverse transcription PCR (RT-PCR) and methylation-specific PCR (MS-PCR) were performed to detect the methylation status of HAPLN1. The mRNA levels of HAPLN1, hsa-miR-204 and $h s a-m i R-218$ were tested by RT-PCR. RNA was reverse transcribed using the PrimeScript ${ }^{\circledR} 1$ st Strand cDNA Synthesis kit (Takara Biotechnology Co., Ltd., Dalian, China) with the following temperature protocol: $30^{\circ} \mathrm{C}$ for $10 \mathrm{~min}, 42^{\circ} \mathrm{C}$ for $60 \mathrm{~min}$ and $95^{\circ} \mathrm{C}$ for $5 \mathrm{~min}$. The $\mathrm{SYBR}{ }^{\circledR}$ Premix Ex Taq $^{\mathrm{TM}}$ kit (Takara Biotechnology Co., Ltd.) and the Applied Biosystems $^{\text {TM }}$ QuantStudio $^{\text {TM }} 5$ Real-Time PCR System (Applied Biosystems; Thermo Fisher Scientific, Inc.) were used to conduct PCR, according to the manufacturer's protocols. DNA methylation modification was performed using an EZ-DNA Methylation-Gold kit ${ }^{\mathrm{TM}}$ (Zymo Research Corp., Irvine, CA, USA), according to the manufacturer's protocols. All the primers were designed and synthesized by Takara Biotechnology Co., Ltd. The MSP primers of HAPLN1 were as follows: Forward, 3'-AGGAGAATTTTTTTGGTGACG T-5' and reverse, 3'-CTA AAAATCAAATAAAACTAA CGCT-5' (210 bp); and the RT-PCR primers were as follows: HAPLN1 forward, 3'-TGGTGAGAAAGTGCCTCCTT-5' 
Table I. The top 20 most significant differentially expressed genes in clear cell renal cell carcinoma samples compared with normal kidney samples.

\begin{tabular}{|c|c|c|c|c|c|}
\hline Gene & Log FC & Mean expression & $\mathrm{t}$ & $\mathrm{P}$-value & $|\Delta \beta|$ \\
\hline NDUFA4L2 & -4.013 & 7.400 & -20.749 & $1.81 \times 10^{-6}$ & 13.385 \\
\hline HK2 & -3.156 & 4.706 & -20.558 & $1.81 \times 10^{-6}$ & 13.307 \\
\hline PCSK6 & -3.022 & 6.983 & -21.826 & $1.81 \times 10^{-6}$ & 13.800 \\
\hline TMEM213 & 5.025 & 4.467 & 19.505 & $2.57 \times 10^{-6}$ & 12.862 \\
\hline NPHS2 & 4.386 & 5.637 & 18.708 & $3.36 \times 10^{-6}$ & 12.500 \\
\hline DMRT2 & 3.306 & 3.416 & 18.248 & $3.77 \times 10^{-6}$ & 12.282 \\
\hline BHLHE41 & -3.750 & 5.475 & -16.519 & $8.56 \times 10^{-6}$ & 11.385 \\
\hline SLC47A2 & 4.148 & 5.832 & 16.690 & $8.56 \times 10^{-6}$ & 11.479 \\
\hline SFRP1 & 2.895 & 5.473 & 16.042 & $1.05 \times 10^{-5}$ & 11.115 \\
\hline AQP6 & 2.851 & 4.375 & 15.661 & $1.24 \times 10^{-5}$ & 10.892 \\
\hline ENO2 & -3.218 & 5.839 & -15.328 & $1.24 \times 10^{-5}$ & 10.690 \\
\hline CNTN1 & 2.911 & 4.580 & 15.444 & $1.24 \times 10^{-5}$ & 10.762 \\
\hline ATP6V0A4 & 3.492 & 4.469 & 15.137 & $1.24 \times 10^{-5}$ & 10.573 \\
\hline TMEM52B & 4.297 & 7.647 & 15.116 & $1.24 \times 10^{-5}$ & 10.560 \\
\hline CLCNKB & 4.451 & 5.645 & 15.133 & $1.24 \times 10^{-5}$ & 10.570 \\
\hline PAH & 6.079 & 5.914 & 14.721 & $1.55 \times 10^{-5}$ & 10.310 \\
\hline NPHS1 & 2.549 & 4.357 & 14.582 & $1.57 \times 10^{-5}$ & 10.220 \\
\hline ATP6V0D2 & 4.435 & 5.594 & 14.548 & $1.57 \times 10^{-5}$ & 10.198 \\
\hline ERBB4 & 3.221 & 3.994 & 14.425 & $1.64 \times 10^{-5}$ & 10.117 \\
\hline MT1G & 5.561 & 7.111 & 14.195 & $1.85 \times 10^{-5}$ & 9.964 \\
\hline
\end{tabular}

and reverse, 3'-TAGCGCTCTTTCTCCTCACC-5' (151 bp); hsa-miR-204 forward, 3'-CAGTGCAGGGTCCGAGGTAT-5' and reverse, 3'-GCTGGAAGGCAAAGGGACGT-5' (180 bp); hsa-miR-218 forward, 3'-CAGTGCAGGGTCCGAGGT AT-5' and reverse, 3'-ATGGTTCCGTCAAGCACCATGG-5' (205 bp); and $\beta$-actin forward, 5'-CTACAATGAGCTGCGT GTGG -3' and reverse, 5'-AGGCATACAGGGACAACACA-3' (308 bp). The thermocycling conditions were as follows: $95^{\circ} \mathrm{C}$ for $5 \mathrm{~min}$; followed by 40 cycles of $95^{\circ} \mathrm{C}$ for $15 \mathrm{sec}, 60^{\circ} \mathrm{C}$ for $30 \mathrm{sec}$, and $72^{\circ} \mathrm{C}$ for $35 \mathrm{sec}$; and a final $5 \mathrm{~min}$ at $72^{\circ} \mathrm{C}$ extension. The $2^{-\Delta \Delta \mathrm{Cq}}$ method was used to calculate the relative expression value of the target gene (16).

Statistical analysis. SPSS version 17.0 (SPSS Inc., Chicago, IL, USA) was used for all statistical analyses, and data are presented as the mean \pm standard deviation. T test was used to compare the two groups and $\mathrm{P}<0.05$ was considered to indicate a statistically significant difference.

\section{Results}

DEGs, DEMs and DMSs. A total of 2,172 (1,089 upregulated and 1,083 downregulated) DEGs, 202 (91 upregulated and 111 downregulated) DEMs and 2,172 (1,305 upregulated and 867 downregulated) DMSs were identified in ccRCC samples compared with normal kidney samples. The top 20 most significantly upregulated/downregulated DEGs, DEMs and DMSs are presented in Tables I, II and III, respectively. The location distribution of DMSs is presented in Fig. 1, and they were primarily located in the gene coding region (31\%) and the intergenic gene region $(22 \%)$.
Enriched GO terms and KEGG pathways. The DEGs were enriched in 1,015 GO terms and 69 KEGG pathways. The top 10 significantly enriched GO terms and KEGG pathways are presented in Tables IV and V, respectively.

Targets of DEMs. The target genes of DEMs were identified in at least 5 algorithms of the miRWalk database and therefore, 10,601 miRNA-gene pairs were obtained. The locations of the target genes and the regulation trends of the miRNA-gene pairs are presented in Fig. 2. More targets were located in the 3'-UTR, fewer in the 5'-UTR and coding domain sequence (CDS) and the majority of miRNA-gene pairs were negatively regulated.

Combination of DEGs, DEMs and DMSs. In total, 143 DEGs involved in DMSs were identified in ccRCC samples compared with normal kidney samples. The gene expression level and DNA methylation level of 45 of these genes exhibited inverse associations (Fig. 3). A total of 851 miRNA-gene pairs were simultaneously involved in DEGs, DEMs and DMS-located genes. Among them, there were 127 miRNA-gene pairs, the genes of which were negatively associated with corresponding DEMs and DMSs. Furthermore, 32 of these miRNA-gene pairs, of which the targeted genes had well-defined genetic locations, are presented in Table VI. The 32 miRNA-gene pairs were composed of 15 genes and 14 miRNAs. HAPLN1 had the most significant differences in expression and was regulated by $h s a-m i R-204$ and $h s a-m i R-218$. Results of the verification are presented in Table VII; HAPLN1 had a lower expression level and a significantly higher methylation level 
Table II. The top 20 most significant differentially expressed microRNA in clear cell renal cell carcinoma samples compared with normal kidney samples.

\begin{tabular}{|c|c|c|c|c|c|}
\hline Gene & Log FC & Mean expression & $\mathrm{t}$ & P-value & $|\Delta \beta|$ \\
\hline hsa-miR-200c & 353.683 & 221.596 & 17.981 & $1.27 \times 10^{-5}$ & 6.195 \\
\hline hsa-miR-141 & 352.019 & 220.817 & 12.544 & $1.27 \times 10^{-5}$ & 4.868 \\
\hline hur_6 & 23789.981 & 42317.538 & 10.142 & 0.001 & 3.852 \\
\hline hsa-miR-342-5p & -19.321 & 60.751 & -9.957 & 0.001 & 3.757 \\
\hline hsa-miR-21 & -36961.351 & 38145.395 & -9.888 & 0.001 & 3.720 \\
\hline hsa-miR-25 & -278.196 & 487.476 & -7.556 & 0.008 & 2.223 \\
\hline hsa-miR-34a & -2270.488 & 1752.008 & -7.214 & 0.009 & 1.951 \\
\hline hsa-miR-15a & -1651.827 & 2258.899 & -7.019 & 0.010 & 1.789 \\
\hline hsa-miR-138 & 34.895 & 59.818 & 6.732 & 0.012 & 1.541 \\
\hline hsa-miR-200b & 1449.075 & 1441.078 & 6.511 & 0.014 & 1.341 \\
\hline hsa-miR-136 & 11.585 & 55.781 & 6.207 & 0.016 & 1.055 \\
\hline hsa-miR-124 & 18.996 & 54.063 & 6.162 & 0.016 & 1.011 \\
\hline hsa-miR-34a & -36.297 & 64.852 & -6.140 & 0.016 & 0.990 \\
\hline hsa-miR-532-5p & 153.632 & 191.707 & 6.050 & 0.016 & 0.901 \\
\hline hsa-miR-342-3p & -357.194 & 500.970 & -5.958 & 0.016 & 0.809 \\
\hline hsa-miR-28-3p & -5.341 & 48.193 & -5.938 & 0.016 & 0.789 \\
\hline hsa-miR-30a & 8011.852 & 8679.002 & 5.902 & 0.016 & 0.752 \\
\hline hsa-miR-193a-5p & -24.702 & 73.083 & -5.799 & 0.016 & 0.647 \\
\hline hsa-miR-362-3p & 120.012 & 182.590 & 5.745 & 0.016 & 0.591 \\
\hline hsa-miR-629 & -4.148 & 46.205 & -5.698 & 0.016 & 0.542 \\
\hline
\end{tabular}

Table III. The top 20 most significant differentially methylated sites in clear cell renal cell carcinoma samples compared with normal kidney samples.

\begin{tabular}{|c|c|c|c|c|}
\hline ID_REF & $\Delta \beta$ & P-value & Gene & Location \\
\hline $\operatorname{cg} 13008315$ & -0.293 & $5.53 \times 10^{-44}$ & & IGS \\
\hline $\operatorname{cg} 22164891$ & -0.473 & $1.30 \times 10^{-41}$ & ZNF217 & TSS200 \\
\hline $\operatorname{cg} 00246451$ & -0.400 & $2.34 \times 10^{-41}$ & ARHGEF2 & TSS1500 \\
\hline $\operatorname{cg} 07166409$ & -0.315 & $1.75 \times 10^{-40}$ & SEMA4C & 5'UTR \\
\hline $\operatorname{cg} 00026222$ & -0.308 & $8.4 \times 10^{-40}$ & & IGS \\
\hline $\operatorname{cg} 19756430$ & -0.273 & $8.85 \times 10^{-39}$ & & IGS \\
\hline $\operatorname{cg} 09228833$ & -0.489 & $1.44 \times 10^{-38}$ & ZNF217 & TSS200 \\
\hline $\operatorname{cg} 19643921$ & -0.257 & $5.50 \times 10^{-37}$ & NUMBL & TSS1500 \\
\hline $\operatorname{cg} 01287592$ & -0.214 & $6.71 \times 10^{-37}$ & DENND3 & 5'UTR \\
\hline $\operatorname{cg} 04312358$ & -0.259 & $1.08 \times 10^{-36}$ & NUMBL & TSS1500 \\
\hline cg09029902 & -0.480 & $1.09 \times 10^{-36}$ & ZNF217 & 5'-UTR; 1stExon \\
\hline $\operatorname{cg} 20979153$ & -0.372 & $1.21 \times 10^{-36}$ & ZNF217 & TSS200 \\
\hline $\operatorname{cg} 08909806$ & -0.245 & $1.22 \times 10^{-36}$ & TSPO & 5'UTR \\
\hline $\operatorname{cg} 27107144$ & -0.211 & $2.19 \times 10^{-36}$ & AES & Body \\
\hline cg07797853 & -0.203 & $1.54 \times 10^{-35}$ & & IGS \\
\hline $\operatorname{cg} 13266096$ & -0.328 & $2.05 \times 10^{-35}$ & MTA2 & Body \\
\hline $\operatorname{cg} 11588197$ & -0.384 & $3.17 \times 10^{-35}$ & ETS1 & Body \\
\hline $\operatorname{cg} 27638217$ & -0.312 & $4.23 \times 10^{-35}$ & & IGS \\
\hline $\operatorname{cg} 08995609$ & -0.374 & $1.19 \times 10^{-34}$ & RIN1 & TSS200 \\
\hline $\operatorname{cg} 06349174$ & -0.211 & $1.58 \times 10^{-34}$ & STIM1 & 1stExon; 5'UTR \\
\hline
\end{tabular}

in ccRCC tissues than in adjacent non-cancerous tissues $(\mathrm{P}<0.0001)$; the expression of $h s a-m i R-204$ and $h s a-m i R-218$ was significantly higher in ccRCC tissues than in adjacent non-cancerous tissues $(\mathrm{P}<0.0001)$. 
Table IV. The top 10 significantly enriched GO terms of differentially expressed genes.

\begin{tabular}{llrr}
\hline Category & & \multicolumn{1}{c}{ Term } & Count \\
\hline GOTERM_CC_5 & GO:0044459 plasma membrane part & 504 & $6.33 \times 10^{-41}$ \\
GOTERM_CC_5 & GO:0070062 extracellular exosome & 510 & $6.68 \times 10^{-35}$ \\
GOTERM_CC_5 & GO:0031226 intrinsic component of plasma membrane & 335 & $3.50 \times 10^{-28}$ \\
GOTERM_CC_5 & GO:0005887 integral component of plasma membrane & 325 & $5.01 \times 10^{-28}$ \\
GOTERM_BP_5 & GO:0006811 ion transport & 280 & $3.33 \times 10^{-23}$ \\
GOTERM_BP_5 & GO:0043436 oxoacid metabolic process & 185 & 184 \\
GOTERM_BP_5 & GO:0019752 carboxylic acid metabolic process & 81 & $3.05 \times 10^{-22}$ \\
GOTERM_CC_5 & GO:0009897 external side of plasma membrane & 89 & $9.78 \times 10^{-20}$ \\
GOTERM_CC_5 & GO:0016324 apical plasma membrane & 194 & $4.76 \times 10^{-19}$ \\
GOTERM_CC_5 & GO:0098590 plasma membrane region & $1.48 \times 10^{-18}$ \\
\hline
\end{tabular}

GO, gene ontology.

Table V. The top 10 significantly enriched KEGG pathways of differentially expressed genes.

\begin{tabular}{llll}
\hline Category & \multicolumn{1}{c}{ Term } & Count & P-value \\
\hline KEGG_PATHWAY & hsa05332:Graft-versus-host disease & 21 & $2.23 \times 10^{-10}$ \\
KEGG_PATHWAY & hsa05150:Staphylococcus aureus infection & 27 & $4.61 \times 10^{-10}$ \\
KEGG_PATHWAY & hsa04940:Type I diabetes mellitus & 22 & $9.44 \times 10^{-9}$ \\
KEGG_PATHWAY & hsa05323:Rheumatoid arthritis & 33 & $2.66 \times 10^{-8}$ \\
KEGG_PATHWAY & hsa04145:Phagosome & 47 & $2.72 \times 10^{-8}$ \\
KEGG_PATHWAY & hsa05330:Allograft rejection & 20 & $2.76 \times 10^{-8}$ \\
KEGG_PATHWAY & hsa05322:Systemic lupus erythematosus & 41 & $2.74 \times 10^{-7}$ \\
KEGG_PATHWAY & hsa04978:Mineral absorption & 26 & $3.97 \times 10^{-7}$ \\
KEGG_PATHWAY & hsa03320:PPAR signaling pathway & 42 & $5.06 \times 10^{-7}$ \\
KEGG_PATHWAY & hsa04514:Cell adhesion molecules (CAMs) & $5.25 \times 10^{-7}$
\end{tabular}

KEGG, Kyoto Encyclopedia of Genes and Genomes.

\section{Discussion}

Genetic variations are associated with the occurrence and development of RCC. miRNAs regulate gene expression and serve an important role in the development of cancer. The methylation status of certain genes is associated with cancer development and metastatic recurrence in ccRCC. In the present study, the mRNA and miRNA expression profiles, as well as the methylation profiles, were analyzed. A total of 2,172 DEGs, 202 DEMs and 2,172 DMSs were identified in ccRCC samples compared with normal kidney samples. The DEGs were enriched in 1,015 GO terms, and the majority of them were associated with the plasma membrane, extracellular exosome and material transport, including the plasma membrane part, extracellular exosome and ion transport (Table IV). Plasma membrane part was the most significant GO term for the DEGs. Plasma membrane part is a cellular component term, which participates in regulating DNA methylation and the mechanism of glioma (17-19). Human plasma membrane-associated sialidase (NEU3), an important cellular component of cell membrane part, serves crucial roles in the regulation of

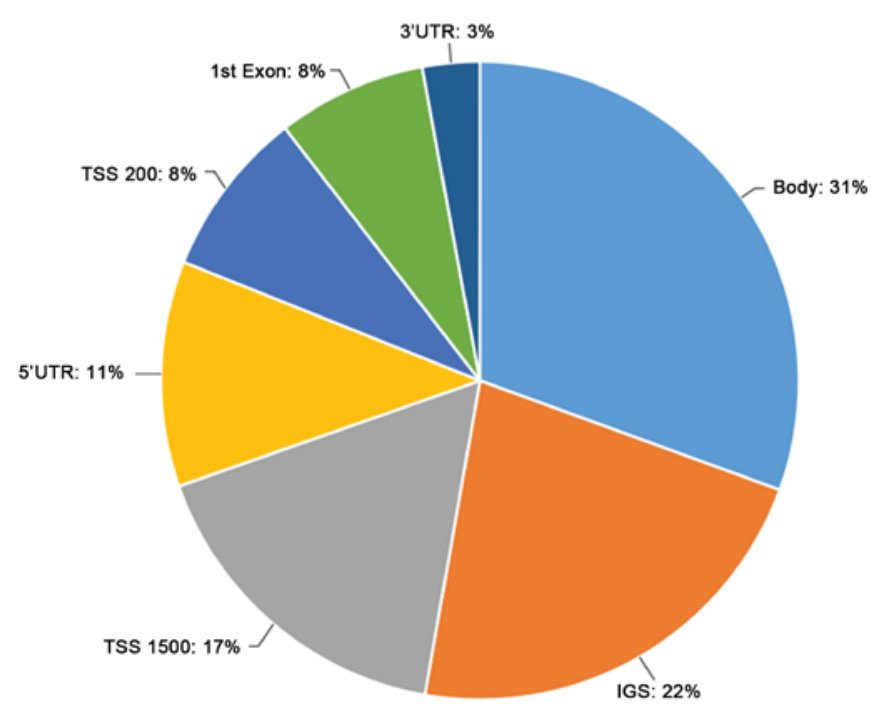

Figure 1. The location distribution of DMSs in genes. The body indicates the gene coding region. TSS200, the $200 \mathrm{bp}$ upstream of the transcription start site; TSS1500, the 1,500 bp upstream of the transcription start site; 5'-UTR, the 5'-untranslated region; 1stExon, the first exon region; 3'-UTR, the 3'-untranslated region; IGS, intergenic gene region; DMSs, the differentially methylated sites. 


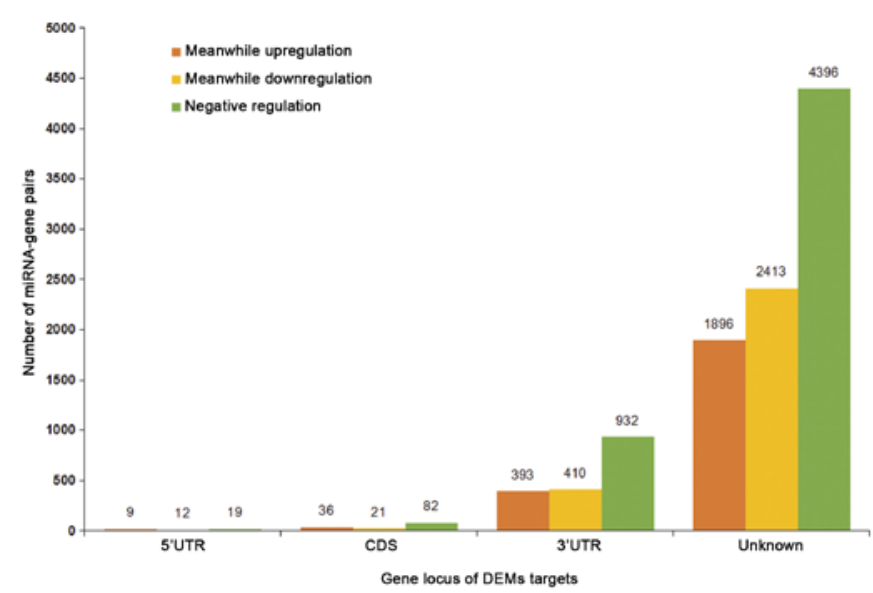

Figure 2. The location distribution of the DEMs targets and the number of miRNA-gene pairs. Upregulation indicates that the DEMs and their targets were upregulated; Downregulation indicates that the DEMs and their targets were downregulated; Negative regulation indicates that the DEMs and their targets were inversely associated. DEMs, differentially expressed miRNAs; 5'-UTR, the 5'-untranslated region; 3'-UTR, the 3'-untranslated region; Unknown, the unknown or undiscovered region; CDS, coding sequence.

cell surface functions. Ueno et al (20) reported that NEU3 was upregulated in RCC and promoted interleukin-6-induced apoptosis suppression and cell motility. Tringali et al (21) demonstrated a crucial role of NEU3 in RCC malignancy by acting as a key regulator of the $\beta 1$ integrin-recycling pathway and FAK/Akt signaling. Therefore, the cellular component term of plasma membrane part was associated with the progression of RCC. Furthermore, the DEGs were enriched in 69 KEGG pathways, including graft-versus-host disease, staphylococcus aureus infection, type I diabetes mellitus and rheumatoid arthritis. Graft-versus-host disease (GvHD) was the most significant pathway. GvHD is a medical complication following the receipt of transplanted tissue from a genetically different person. It is commonly associated with stem cell transplant (bone marrow transplant), but the term also applies to other forms of tissue graft. A previous study revealed a reduced rate of GvHD during cyclophosphamide-using non-myeloablative cell therapy against renal cancer (22). Another study indicated that the graft vs. tumor reactivity following allogeneic stem cell transplantation may be unavoidably associated with GvHD in patients with RCC (23). Additionally, Massenkeil et al (24) reported that non-myeloablative stem cell transplantation in metastatic renal cell carcinoma delayed GvHD. In the present study, we hypothesized that GvHD may serve certain roles in the pathogenesis of RCC and that further functional studies were required.

Following combination analysis of DEGs, DEMs and DMSs, HAPLN1 was one of the DEGs that was negatively regulated by their corresponding targeted DEMs and DMSs, and it had well-defined genetic locations. Furthermore, HAPLN1 exhibited the most pronounced differences in expression, and was negatively regulated by $h s a-m i R-204$ and $h s a-m i R-218$. Table VI indicates that $h s a-m i R-204$ and hsa-miR-218 targeted the 3'-UTR of HAPLN1. It is well known that miRNAs block the transcription of their target genes when they target the 3'-UTR (25). In the present study, the expression of HAPLNI was negatively associated with the expression

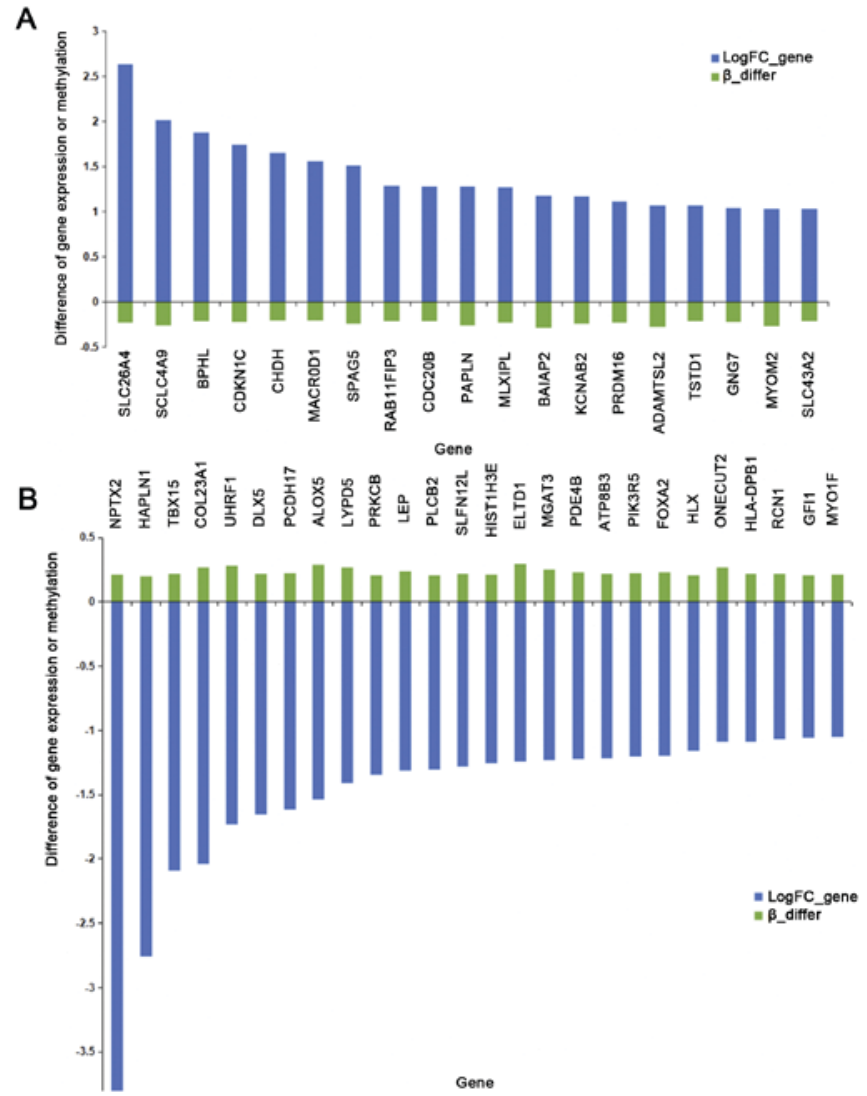

Figure 3 . The 45 differentially expressed and methylated genes with inversely associated gene expression and DNA methylation levels in ccRCC samples compared with normal kidney samples. (A) The genes were upregulated, differentially expressed and hypomethylated. (B) The genes were downregulated, differentially expressed and hypermethylated. ccRCC, cell renal cell carcinoma; $\operatorname{LogFC}$ _gene, fold-change in gene differential expression; $\beta$ _differ, fold-change in gene methylation level.

of hsa-miR-204 and hsa-miR-218. Additionally, the methylation site of HAPLN1 is located in the transcriptional start site $1,500 \mathrm{bp}$ (TSS1500) region. In this region, gene methylation may lead to deletion or downregulation of gene expression. In the present study, the expression of $H A P L N 1$ was negatively associated with the methylation level. Furthermore, HAPLN1 and $h s a-m i R-204$ were the most significantly different gene and DEM, respectively (Table VI). HAPLN1 is a protein that in humans is encoded by the HAPLNI gene. HAPLN1 is an extracellular matrix component serving an important role in heart development, and is associated with cerebral creatine deficiency syndrome and fracture. It was reported that overexpression of HAPLN1 and its SP-IgV domain increased the tumorigenic properties of mesothelioma (26). Yau et al (27) identified HAPLN1 as a novel prognostic gene candidate to predict the outcome of breast cancer. Mebarki et al (28) proved that HAPLN1 reflected a signaling network leading to stemness, mesenchymal commitment and progression in hepatocellular carcinoma. The present study, revealed that HAPLN1 had a low expression level and a high methylation level in ccRCC tissues (Table VII), which may be involved in the occurrence of ccRCC. Hsa-miR-204 was identified to be highly expressed in lymphocytic leukemia, and it was differentially expressed during the progression of recurrence in hepatocellular carcinoma and gastric cancer (29-31). Hsa-miR-218 was reported to 
Table VI. The 32 microRNA-gene pairs, the target genes of which were negatively regulated by corresponding differentially expressed miRNA and differentially methylated sites, and had well-defined genetic locations.

\begin{tabular}{|c|c|c|c|c|c|c|}
\hline MicroRNA & Gene & MiRNA_logFC & Gene_LogFC & Beta_diff & Gene_locus & Methy_loc \\
\hline hsa-miR-204 & HAPLN1 & 3179.242 & -2.756 & 0.202 & 3'-UTR & TSS 1500 \\
\hline hsa-miR-218 & HAPLN1 & 189.092 & -2.756 & 0.202 & 3'-UTR & TSS 1500 \\
\hline hsa-miR-106b & SLC26A4 & -519.831 & 2.631 & -0.227 & 3'-UTR & TSS1500; Body \\
\hline hsa-miR-106b & BPHL & -519.831 & 1.878 & -0.211 & 3'-UTR & Body \\
\hline hsa-miR-124 & DLX5 & 18.996 & -1.652 & 0.221 & 3'-UTR & Body \\
\hline hsa-miR-125a-5p & ALOX5 & 125.869 & -1.537 & 0.288 & 3'-UTR & Body \\
\hline hsa-miR-183 & ALOX5 & 10.550 & -1.537 & 0.288 & 3'-UTR & Body \\
\hline hsa-miR-125a-5p & LEP & 125.869 & -1.314 & 0.236 & 3'-UTR & TSS 1500 \\
\hline hsa-miR-29b & LEP & 1101.186 & -1.314 & 0.236 & 3'-UTR & TSS 1500 \\
\hline hsa-miR-29c & LEP & 1699.774 & -1.314 & 0.236 & 3'-UTR & TSS 1500 \\
\hline hsa-miR-30b & LEP & 1746.324 & -1.314 & 0.236 & 3'-UTR & TSS 1500 \\
\hline hsa-let-7a & PLCB2 & 4972.969 & -1.303 & 0.206 & 3'-UTR & Body \\
\hline hsa-let-7c & PLCB2 & 580.187 & -1.303 & 0.206 & 3'-UTR & Body \\
\hline hsa-let-7f & PLCB2 & 4506.520 & -1.303 & 0.206 & 3'-UTR & Body \\
\hline hsa-let-7g & PLCB2 & 531.008 & -1.303 & 0.206 & 3'-UTR & Body \\
\hline hsa-miR-204 & PDE4B & 3179.242 & -1.224 & 0.232 & 3'-UTR & TSS200;TSS1500 \\
\hline hsa-miR-125a-5p & PIK3R5 & 125.869 & -1.203 & 0.222 & 3'-UTR & TSS200 \\
\hline hsa-miR-29b & PIK3R5 & 1101.186 & -1.203 & 0.222 & 3'-UTR & TSS200 \\
\hline hsa-miR-29c & PIK3R5 & 1699.774 & -1.203 & 0.222 & 3'-UTR & TSS200 \\
\hline hsa-miR-337-5p & FOXA2 & 6.426 & -1.198 & 0.233 & 3'-UTR & Body; 3'UTR \\
\hline hsa-let-7a & HLX & 3179.242 & -2.756 & 0.202 & 3'-UTR & 3'-UTR \\
\hline hsa-let-7c & HLX & 189.092 & -2.756 & 0.202 & 3'-UTR & 3'-UTR \\
\hline hsa-let-7f & HLX & -519.831 & 2.631 & -0.227 & 3'-UTR & 3'-UTR \\
\hline hsa-let-7g & HLX & -519.831 & 1.878 & -0.211 & 3'-UTR & 3'-UTR \\
\hline hsa-miR-30b & HLX & 1746.324 & -1.157 & 0.206 & 3'-UTR & 3'-UTR \\
\hline hsa-miR-125a-5p & ONECUT2 & 125.869 & -1.087 & 0.271 & CDS & 1stExon \\
\hline hsa-miR-124 & HLA-DPB 1 & 18.996 & -1.084 & 0.220 & 3'-UTR & Body \\
\hline hsa-miR-106b & ADAMTSL2 & -519.831 & 1.073 & -0.277 & 3'-UTR & Body \\
\hline hsa-let-7a & MYO1F & 4972.969 & -1.050 & 0.212 & CDS & Body \\
\hline hsa-let-7c & MYO1F & 580.187 & -1.050 & 0.212 & CDS & Body \\
\hline hsa-let-7f & MYO1F & 4506.520 & -1.050 & 0.212 & CDS & Body \\
\hline
\end{tabular}

Table VII. Results of methylation-specific polymerase chain reaction and reverse transcription-polymerase chain reaction.

\begin{tabular}{lcccc}
\hline Group & HAPLN1-methy & HAPLN1-mRNA & Hsa-miR-204 & Hsa-miR-218 \\
\hline ccRCC tissues & $4.228 \pm 1.061$ & $0.466 \pm 0.512$ & $4.377 \pm 1.057$ & $4.627 \pm 1.189$ \\
Adjacent tissues & $1.034 \pm 0.024$ & $1.064 \pm 0.671$ & $1.037 \pm 0.021$ & $1.029 \pm 0.020$ \\
P-value & $<0.0001$ & $<0.0001$ & $<0.0001$ & $<.0001$ \\
T & 9.69 & -6.06 & 15.23 & 14.93 \\
\hline
\end{tabular}

$\mathrm{n}=10$. ccRCC, clear cell renal cell carcinoma.

serve an important role in the proliferation and metastasis of colon carcinoma (32). Additionally, $h s a-m i R-218$ may inhibit the multidrug resistance of gastric cancer cells (33). In the present study, hsa-miR-204 and $h s a-m i R-218$ were proven to be highly expressed in ccRCC tissues, and may serve certain roles in the pathogenesis of RCC by targeting HAPLN1.
In conclusion, the present study identified certain biomarkers of RCC by combination analysis of a mRNA expression profile, a miRNA expression profile and a methylation profile, including HAPLN1, hsa-miR-204 and hsa-miR-218. Additionally, the cellular component of plasma membrane part and the pathway of GvHD may be involved in 
the pathogenesis of RCC. However, there are certain limitations to the present study. The sample size was small in the profiles and verification, and therefore the identified genes and miRNAs may have greater specificity and less universality. The biomarkers screened in the present study provided an indication to study the pathogenesis of RCC. Additionally, HAPLN1, hsa-miR-204 and $h s a-m i R-218$ require further investigation in larger samples to elucidate their exact function and clinical significance.

\section{Acknowledgements}

Not applicable.

\section{Funding}

No funding was received.

\section{Availability of data and materials}

All data generated or analyzed during the present study are included in this published article.

\section{Authors' contributions}

YX designed the experiments. ZW and ZZ performed data analysis. ZW and CZ interpreted the data and wrote the manuscript. ZW and YX discussed the results and revised the manuscript. All authors contributed to discussions regarding the results and the manuscript. All authors have read and approved the final manuscript.

\section{Ethics approval and consent to participate}

Written informed consent was obtained when the patients were accepted by the Second Hospital of Tianjin Medical University. All procedures were performed in accordance with the ethical standards of the institutional and/or national research committee.

\section{Consent for publication}

Consent for publication was obtained from all patients.

\section{Competing interests}

The authors declare that they have no competing interests.

\section{References}

1. Hsieh JJ, Purdue MP, Signoretti S, Swanton C, Albiges L, Schmidinger M, Heng DY, Larkin J and Ficarra V: Renal cell carcinoma. Nat Rev Dis Primers 3: 17009, 2017.

2. Protzel C, Maruschke M and Hakenberg OW: Epidemiology, aetiology, and pathogenesis of renal cell carcinoma. Eur Urol Supp 11: 52-59, 2012.

3. Baek M, Jung JY, Kim JJ, Park KH and Ryu DS: Characteristics and clinical outcomes of renal cell carcinoma in children: a single center experience. Int J Urol 17: 737-740, 2010.

4. Häggström C, Rapp K, Stocks T, Manjer J, Bjørge T, Ulmer H, Engeland A, Almqvist M, Concin H, Selmer R, et al: Correction: Metabolic factors associated with risk of renal cell carcinoma. PloS One 8: e57475, 2013.
5. Trpkov K, Hes O, Agaimy A, Bonert M, Martinek P, Magi-Galluzzi C, Kristiansen G, Lüders C, Nesi G, Compérat E, et al: Fumarate hydratase-deficient renal cell carcinoma is strongly correlated with fumarate hydratase mutation and hereditary leiomyomatosis and renal cell carcinoma syndrome. Am J Surg Pathol 40: 865, 2016.

6. Matsuura K, Nakada C, Mashio M, Narimatsu T, Yoshimoto T, Tanigawa M, Tsukamoto Y,Hijiya N, Takeuchi I, Nomura T, et al: Downregulation of SAV1 plays a role in pathogenesis of high-grade clear cell renal cell carcinoma. BMC Cancer 11: 523, 2011.

7. White NM, Bao TT, Grigull J, Youssef YM, Girgis A, Diamandis M, Fatoohi E, Metias M, Honey RJ, Stewart R, et al: miRNA profiling for clear cell renal cell carcinoma: Biomarker discovery and identification of potential controls and consequences of miRNA dysregulation. J Urol 186: 1077-1083, 2011.

8. Fedorko M, Pacik D, Wasserbauer R, Juracek J, Varga G, Ghazal M and Nussir MI: microRNAs in the pathogenesis of renal cell carcinoma and their diagnostic and prognostic utility as cancer biomarkers. Int J Biol Markers 31: e26-e 37, 2015.

9. Tsai HC and Baylin SB: Cancer epigenetics: Linking basic biology to clinical medicine. Cell Res 21: 502-517, 2011.

10. Ricketts CJ, Morris MR, Gentle D, Brown M, Wake N, Woodward ER, Clarke N, Latif F and Maher ER: Genome-wide $\mathrm{CpG}$ island methylation analysis implicates novel genes in the pathogenesis of renal cell carcinoma. Epigenetics 7: 278-290, 2012.

11. Wang X, Chen X, Han W, Ruan A, Chen L, Wang R, Xu Z, Xiao P3, Lu X, Zhao Y, et al: miR-200c targets CDK2 and suppresses tumorigenesis in renal cell carcinoma. Mol Cancer Res 13: 1567-1577, 2015.

12. Wei JH,Haddad A,Wu KJ,ZhaoHW,KapurP,Zhang ZL,ZhaoLY, Chen ZH, Zhou YY, Zhou JC, et al: A CpG-methylation-based assay to predict survival in clear cell renal cell carcinoma. Nat Commun 6: 8699, 2015.

13. Wiberg AO,Liu L, Tong Z,Myslivets E, Ataie V,KuoBP,Alic Nand Radic S: Photonic preprocessor for analog-to-digital-converter using a cavity-less pulse source. Opt Express 20: B419-B427, 2012.

14. Dennis G Jr, Sherman BT, Hosack DA, Yang J, Gao W, Lane HC and Lempicki RA: DAVID: Database for annotation, visualization, and integrated discovery. Genome Biol 4: P3, 2003.

15. Dweep H, Sticht C, Pandey P and Gretz N: miRWalk-database: Prediction of possible miRNA binding sites by 'walking' the genes of three genomes. J Biomed Inform 44: 839-847, 2011.

16. Livak KJ and Schmittgen TD: Analysis of relative gene expression data using real-time quantitative PCR and the 2(-Delta Delta C(T)) method. Methods 25: 402-408, 2001.

17. Nautiyal S, Carlton VE, Lu Y, Ireland JS, Flaucher D, Moorhead M, Gray JW, Spellman P, Mindrinos M, Berg P and Faham M: High-throughput method for analyzing methylation of CpGs in targeted genomic regions. Proc Natl Acad Sci USA 107: 12587-12592, 2010.

18. Gao YF, Shu Y, Yang L, He YC, Li LP, Huang G, Li HP and Jiang Y: A graphic method for identification of novel glioma related genes. Biomed Res Int 2014: 891945, 2014.

19. Denham J, O'Brien BJ, Harvey JT and Charchar FJ: Genome-wide sperm DNA methylation changes after 3 months of exercise training in humans. Epigenomics 7: 717-731, 2015.

20. Ueno S, Saito S, Wada T, Yamaguchi K, Satoh M, Arai Y and Miyagi T: Plasma membrane-associated sialidase is up-regulated in renal cell carcinoma and promotes interleukin-6-induced apoptosis suppression and cell motility. J Biol Chem 281: 7756-7764, 2006

21. Tringali C, Lupo B, Silvestri I, Papini N, Anastasia L, Tettamanti $G$ and Venerando B: The plasma membrane sialidase NEU3 regulates the malignancy of renal carcinoma cells by controlling $\beta 1$ integrin internalization and recycling. J Biol Chem 287: 42835-42845, 2012.

22. Eto M, Harano M, Tatsugami K, Harada M, Kamiryo Y, Kiyoshima K, Hamaguchi M, Tsuneyoshi M, Yoshikai Y and Naito S: Cyclophosphamide-using nonmyeloablative allogeneic cell therapy against renal cancer with a reduced risk of graft-versus-host disease. Clin Cancer Res 13: 1029-1035, 2007.

23. van Bergen CA, Verdegaal EME, Honders MW, Hoogstraten C, Steijn-van Tol AQ, de Quartel L, de Jong J, Meyering M, Falkenburg JH, Griffioen M and Osanto S: Durable remission of renal cell carcinoma in conjuncture with graft versus host disease following allogeneic stem cell transplantation and donor lymphocyte infusion: Rule or exception? PloS One 9: e85198, 2014. 
24. Massenkeil G, Roigas J, Nagy M, Wille A, Stroszczynski C, Mapara MY, Loening S, Dörken B and Arnold R: Nonmyeloablative stem cell transplantation in metastatic renal cell carcinoma: Delayed graft-versus-tumor effect is associated with chimerism conversion but transplantation has high toxicity. Bone Marrow Transplant 34: 309-316, 2004.

25. Endale Ahanda ML, Fritz ER, Estellé J, Hu ZL, Madsen O, Groenen MA, Beraldi D, Kapetanovic R, Hume DA, Rowland RR, et al: Prediction of altered 3'-UTR miRNA-binding sites from RNA-Seq data: The swine leukocyte antigen complex (SLA) as a model region. Plos One 7: e48607, 2012.

26. Ivanova AV, Goparaju CM, Ivanov SV, Nonaka D, Cruz C, Beck A, Lonardo F, Wali A and Pass HI: Protumorigenic role of HAPLN1 and its IgV domain in malignant pleural mesothelioma. Clin Cancer Res 15: 2602-2611, 2009.

27. Yau C, Esserman L, Moore DH, Waldman F, Sninsky J and Benz CC: A multigene predictor of metastatic outcome in early stage hormone receptor-negative and triple-negative breast cancer. Breast Cancer Res 12: R85, 2010.

28. Mebarki S, Désert R, Sulpice L, Sicard M, Desille M, Canal F, Dubois-Pot Schneider H, Bergeat D, Turlin B, Bellaud P, et al: De novo HAPLN1 expression hallmarks Wnt-induced stem cell and fibrogenic networks leading to aggressive human hepatocellular carcinomas. Oncotarget 7: 39026-39043, 2016.

29. Zanette DL, Rivadavia F, Molfetta GA, Barbuzano FG, Proto-Siqueira R, Silva WA Jr, Falcão RP and Zago MA: miRNA expression profiles in chronic lymphocytic and acute lymphocytic leukemia. Braz J Med Biol Res 40: 1435-1440, 2007.

30. Yang Z, Miao R, Li G, Wu Y, Robson SC, Yang X, Zhao Y, Zhao $\mathrm{H}$ and Zhong Y: Identification of recurrence related microRNAs in hepatocellular carcinoma after surgical resection. Int J Mol Sci 14: 1105-1118, 2013.
31. Wang XW, Wu Y, Wang D and Qin ZF: microRNA network analysis identifies key microRNAs and genes associated with precancerous lesions of gastric cancer. Genet Mol Res 13: 8695-8703, 2014.

32. Jiang B, Liu GW and Xie HH: Expression and its clinical significance of hsa-miR-218 in tissues of colon carcinoma. Med Info, 2010.

33. Zhang XL, Shi HJ, Wang JP, Tang HS and Cui SZ: miR-218 inhibits multidrug resistance (MDR) of gastric cancer cells by targeting hedgehog/smoothened. Int J Clin Exp Pathol 8: 6397-6406, 2015.

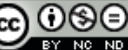

This work is licensed under a Creative Commons Attribution-NonCommercial-NoDerivatives 4.0 International (CC BY-NC-ND 4.0) License. 